\title{
Targeted Osteomyelitis Management Using Metallic Nanoparticles
}

\author{
Shahnaz Majid Qadri, Tahir Abdulrehman, Yousef Haik \\ Hamad Bin Khalifa University, College of Science and Engineering \\ Qatar Foundation, Education City, Doha, Qatar \\ sqadri@hbku.edu.qa; tabdulrahman@hbku.edu.qa
}

\section{Extended Abstract}

Osteomyelitis is a serious bone infection which can be caused by the bacteria Staphylococcus aureus. The bacteria can survive inside osteoblast cells and overcome the effect of the immune system as well as various antibiotics. Silver-CopperBoron $(\mathrm{AgCuB})$ nanoparticles were found to be effective against $S$. aureus in both in vitro and in vivo studies. For accurate therapy, targeting the site of infection is essential. In this study, for targeted bone therapy, AgCuB nanoparticles are coated with bone targeting antibodies (Anti-OB-Cadherin antibody that are specific to osteoblast cells) or bone targeting molecules or anti-S. aureus antibodies to increase the action of the antimicrobial nanoparticles specifically in the bone tissue and is evaluated by in vitro studies and using in vivo mice model.

Staphylococcus aureus is one of the primary causes of bone infection and its persistence in osteoblast cells leads to relapse and acute osteomyelitis [1]. S. aureus survives inside mammalian cells and remains protected from the immune system. Due to the sheltered environment, such acute or chronic infection is difficult to manage [2]. Moreover, this intracellular bacterial reservoir remains protected from a wide range of antibiotics including vancomycin [3]. Earlier we have shown the antimicrobial activity of Silver-Copper-Boron $(\mathrm{AgCuB})$ nanoparticles and also that an $\mathrm{AgCuB}$ dose of 1 $\mathrm{mg} / \mathrm{kg}$ body weight is safe in mice [4]. Also, we have shown that $\mathrm{AgCuB}$ nanoparticles reduced more than $99 \%$ reduction of infection when an $\mathrm{AgCuB}$ dose of $1 \mathrm{mg} / \mathrm{kg}$ body weight was introduced intravenously in in vivo mice model [5]. In this study, we aimed to evaluate the targeted antimicrobial activity at the intracellular level in both in vitro and in vivo models. Here, bone targeting or antibody coated $\mathrm{AgCuB}$ antimicrobial nanoparticles were evaluated for their activity in intracellular infection, employing an in vitro osteoblast infection model as well as an in vivo osteomyelitis mice model. Our in vitro osteoblast infection model showed that $1 \mu \mathrm{g} / \mathrm{ml}$ concentration of nascent $\mathrm{AgCuB}$ nanoparticles reduced more than 90\% infection within 24 hours of treatment.

$\mathrm{AgCuB}$ nanoparticles were synthesized by green chemistry and were characterized by scanning electron microscopy. Furthermore, $\mathrm{AgCuB}$ nanoparticles were conjugated with Anti-OB-Cadherin antibodies or anti-S. aureus antibodies labeled with an AF-594 fluorophore for osteoblast cell targeting or S. aureus targeting respectively. Osteoblast cells targeted with AgCuB-Anti-OB-Cadherin (NP-AntiCad) were evaluated for membrane binding by optical fluorescent microscopy. To measure the antimicrobial activity of NP-AntiCad, S. aureus cultures were grown in osteoblast cell culture media with varying concentrations of NP-AntiCad and were incubated for different time periods. Optical density was measured to check the inhibition of $S$. aureus. To measure the antimicrobial activity of NP-AntiCad against internalized $S$. aureus infection, Osteoblast cells were co-cultured with $S$. aureus and then Gentamicin was used to clean the extracellular infection. Co-cultured cells were added with NP-AntiCad and incubated for 24 hours or 48 hours. After incubation cells were washed and harvested by trypsinization and lysed with Triton-X100. The lysate was serially diluted and plated on TS agar plate, incubated overnight and the following day CFU's were enumerated. The in vitro studies showed that targeted $\mathrm{AgCuB}$ nanoparticles could be an effective treatment strategy for osteomyelitis and lead to targeted in vivo studies.

$\mathrm{AgCuB}$ nanoparticles can be used as an alternative for treating osteomyelitis. Targeting the site of infection by using $\mathrm{AgCuB}$ nanoparticles that are linked to bone targeting agents or specific antibodies could help to lower the dose of nanoparticles used and also to accurately target the site of infection. This unique bactericidal nanoparticle may bring new hope to clinical therapies. More studies have to be conducted to accurately target the site of bone infection. 


\section{References:}

[1] M. E. Olson and A. R. Horswill, "Staphylococcus aureus Osteomyelitis: Bad to the bone," Cell Host Microbe, vol. 13, no. 6, pp. 629-631, 2013.

[2] S. M. Lehar et al., "Novel antibody-antibiotic conjugate eliminates intracellular S. aureus," Nature, vol. 527, no. 7578, pp. 323-328, 2015.

[3] Y. Pei, M. F. Mohamed, M. N. Seleem, and Y. Yeo, "Particle engineering for intracellular delivery of vancomycin to methicillin-resistant Staphylococcus aureus (MRSA)-infected macrophages," J. Control. Release, vol. 267, pp. 133$143,2017$.

[4] K. B. Ramadi et al., "Acute systemic exposure to silver-based nanoparticles induces hepatotoxicity and NLRP3dependent inflammation," Nanotoxicology, vol. 10, no. 8, pp. 1061-1074, 2016.

[5] S. Qadri, Y. Haik, E. Mensah-Brown, G. Bashir, M. J. Fernandez-Cabezudo, and B. K. al-Ramadi, "Metallic nanoparticles to eradicate bacterial bone infection," Nanomedicine Nanotechnology, Biol. Med., vol. 13, no. 7, pp. 2241-2250, 2017. 